\title{
Clinical and morphological correlations for transglutaminase 1 gene mutations in autosomal recessive congenital ichthyosis
}

\author{
Elina Laiho ${ }^{1}$, Kirsti-Maria Niemi ${ }^{2}$, Jaakko Ignatius ${ }^{3}$, Juha Kere ${ }^{4,5}$, Aarno Palotie ${ }^{1,6}$ and \\ Ulpu Saarialho-Kere ${ }^{2}$ \\ ${ }^{1}$ Department of Clinical Chemistry and Institute of Biomedicine, University of Helsinki, and Laboratory Department \\ of Helsinki University Central Hospital \\ ${ }^{2}$ Department of Dermatology, Helsinki University Central Hospital, Helsinki \\ ${ }^{3}$ Jorvi Hospital, Department of Clinical Neurophysiology, Espoo \\ ${ }^{4}$ Haartman Institute, Department of Medical Genetics, Finland \\ ${ }^{5}$ Finnish Genome Center, University of Helsinki, Finland \\ ${ }^{6}$ Department of Human Genetics, UCLA School of Medicine, Los Angeles, California, USA
}

\begin{abstract}
Autosomal recessive congenital ichthyosis (ARCI) is a group of inherited disorders of cornification in which progress has recently been made in the identification of pathogenic mechanisms causing the disease. Transglutaminase 1 (TGM 1) has been found as a defective gene in a large fraction of patients with lamellar ichthyosis (LI), a severe inherited scaling disorder of the skin. We have previously performed molecular genetic studies of 38 Finnish ARCI families and found six different mutations in 13 families of $38(34 \%)$. In this study we compared the molecular genetic alterations with clinical and electron microscopic findings of these patients. Families were classified by electron microscopy in ichthyosis congenita (IC) types I, II, III, IV and a non-defined group. TGM 1 gene mutation was found in all of the IC type II and $1 / 3$ of the IC type 1 families. Although electron microscopy is not always used to classify ARCI patients, it can distinguish groups which are parallel with molecular genetic findings. This finding might be useful in the classification of ARCI patients for further linkage studies. Clinically typical phenotype of the TGM1 mutation carrier includes large, thick, brownish scales, but ichthyosis of some of these patients tends to be milder.
\end{abstract}

Keywords: transglutaminase 1; keratinocyte transglutaminase; lamellar ichthyosis; non-bullous congenital ichthyosiform erythroderma; genodermatosis; genotype-phenotype correlation

Correspondence: Aarno Palotie/Elina Laiho, Laboratory of Molecular Genetics, Helsinki University Central Hospital, Haartmaninkatu 4, SF-00029 HYKS, Helsinki, Finland. Tel: + 358947175865; Fax: +358947174001; E-mail: Elina.Laiho@HUCH.fi

Received 4 January 1999; revised 22 March 1999; accepted 7 April 1999 


\section{Introduction}

Autosomal recessive congenital ichthyosis (ARCI), a heterogeneous group of skin diseases, is classically divided into two categories: lamellar ichthyosis (LI) and congenital ichthyosiform erythroderma (CIE). ${ }^{1}$ Patients with LI are often born encased in a collodion membrane which later changes into scaly skin. The degree of desquamation in LI is severe and ectropion of the eyes and alopecia is often present. Scales of the CIE patients are typically thinner and erythroderma is common, although they may also be born as collodion babies. A number of patients do not fill the criteria of either of these groups and thus the clinical classification is often ambiguous. ${ }^{2}$ ARCI patients are often treated with either oral or topical retinoids.

Although most dermatologists group ARCI patients into LI and CIE, also other models for classification of ARCI exist. A classification based on clinical and electron microscopic findings has been presented by Anton-Lamprecht. ${ }^{3}$ In this classification ARCI patients are divided into four ichthyosis congenita (IC) groups.

The molecular background of the LI phenotype has revealed at least two loci which may be altered, ${ }^{4-6}$ but there are also LI patients who do not segregate any of these loci. One of the known loci in chromosome 14 includes the only gene known to be defective in LI, transglutaminase 1 (TGM1) (also called keratinocyte transglutaminase), which encodes an enzyme important in terminal differentiation of keratinocytes. A structure called cornified cell envelope (CE) is formed in the final stages of epidermal differentiation and by electron microscopy, it is seen on the inner side of the plasma membrane as a $7-15 \mathrm{~nm}$ thick structure. ${ }^{7} \mathrm{CE}$ consists of two layers, a protein envelope (about $90 \%$ of CE mass) and a lipid envelope (10\% of mass). ${ }^{8}$ It is thought to protect the uppermost cells against water and water loss, microbes and other harmful agents and to interact with intermediate filaments to strengthen the cytoskeleton. ${ }^{9}$ The function of TGase1 (enzyme product of the $T G M 1$ gene) is to link precursor proteins, eg loricrin, involucrin and small proline rich proteins, together so that $\mathrm{CE}$ is finally formed. ${ }^{10}$

We have previously studied 38 ARCI families which were classified in IC types I, II, III, IV and the nonclassified group by electron microscopy. ${ }^{11}$ Among these, 17 patients from 13 families were found to carry mutations in the TGM 1 gene. Six different mutations were found: Arg-141-His, Arg-142-Cys, Gly-217-Ser, 3349A to G, Val-378-Leu and Arg-395-Leu. In this study we compare the molecular genetic data concerning transglutaminase 1 with the clinical and ultrastructural findings in these ARCI patients.

\section{Patients and Methods}

We studied 49 Finnish ARCI patients from 38 families. The diagnosis of ARCI was based on electron microscopy and clinical findings. Classification to electron microscopic IC types was based purely on the electron microscopic criteria of Anton-Lamprecht ${ }^{3}$ and was done at the Department of Dermatology, Helsinki University Central Hospital, Finland. All these patients have visited the Department of Dermatology several times. Age of onset, clinical presentation and visible granular layer in routine histopathology had excluded ichthyosis vulgaris. Sex and clinical features excluded $\mathrm{X}$-linked ichthyosis and also assessment of steroid sulfatase level was made in unclear cases. None of the patients had neurological symptoms. The status of retinoid treatment at the time of electron-microscopic diagnosis is listed in Table 1.

\section{IC Type I Patients}

Clinically most of the patients have generalised fine scaling ichthyosis with variable erythroderma, but there is clear heterogeneity in the degree of severity among affected individuals. The most common electron-microscopic markers include lipid droplets or rings and lamellar structures, but the markers are not as specific as the findings in other IC types (Figure 1a). ${ }^{12}$

\section{IC Type /I Patients}

IC type II is the most clearly defined of the IC groups. Patients have a clear clinical picture of classical lamellar ichthyosis with large brown scales. Crystalloid structures called cholestrol clefts in the thickened corneal layer are used as an electron-microscopic marker for IC type II and were found in every patient (Figure 1b). ${ }^{13}$

\section{IC Type III Patients}

The ultrastructural markers are elongated membrane structures, abnormal keratinosomes and vesicular complexes in upper epidermal and horny cells (Figure 1c). The clinical picture differs from other types: the onset of ichthyosis is variable, ichthyosis as well as erythema can be patchy or generalised and flexures are typical places for ichthyosis in these patients. ${ }^{14}$

\section{IC Type IV Patients}

There are only few cases of this type of ichthyosis reported. The course of the disease can be lethal in the newborn due to respiratory distress syndrome detected in some cases. Later the clinical picture is mild and follicular hyperkeratosis gives the skin a goose skin-like appearance. In electron microscopy the packages of trilaminar membrane structures in upper epidermal and horny cells are typical for this type of ichthyosis and were found in both our patients (Figure 1d). ${ }^{15}$

\section{Electron Microscopy}

Biopsy specimens from the upper arm and other parts of lesional skin were fixed in a routine manner during the years 


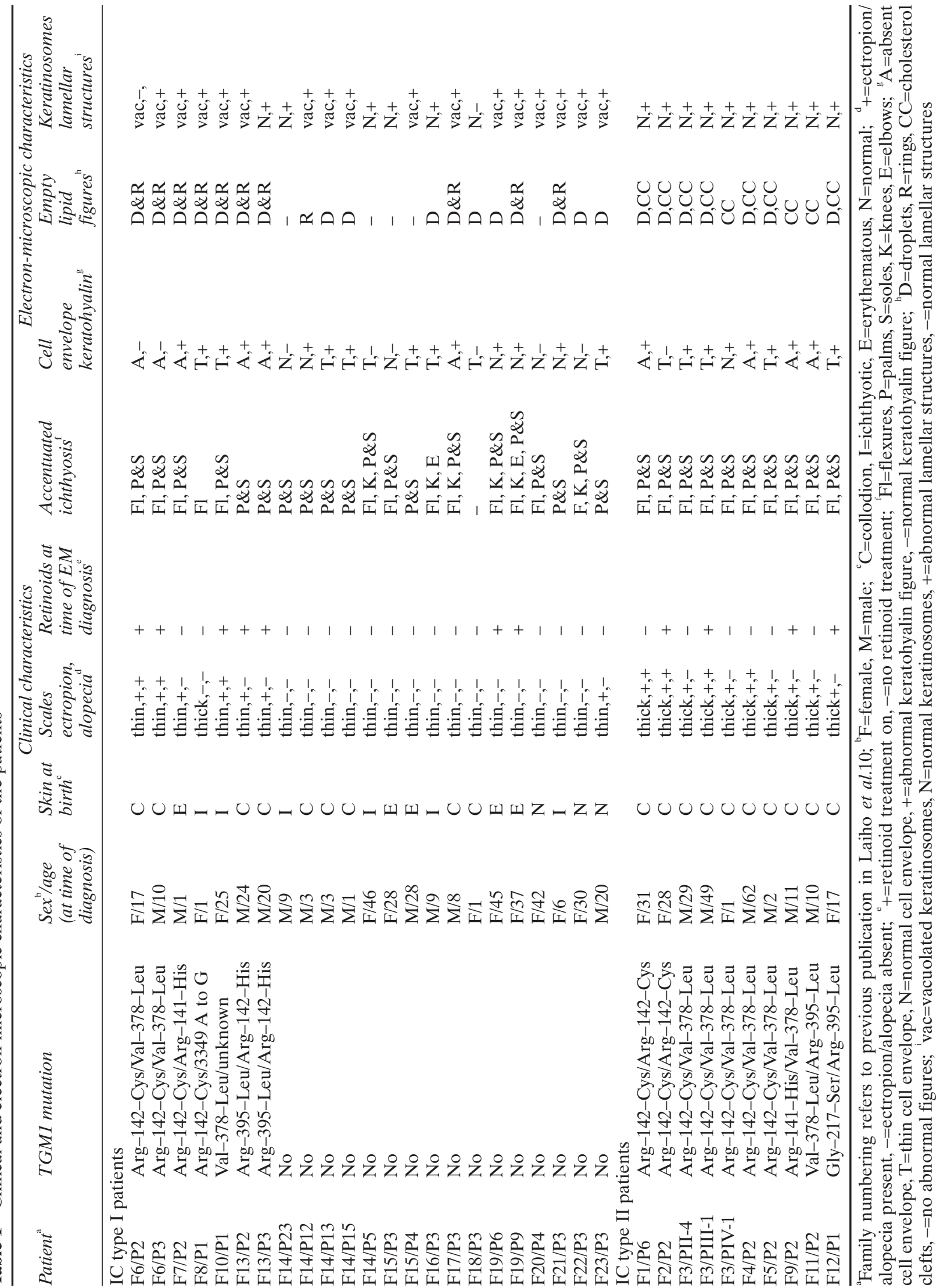


A

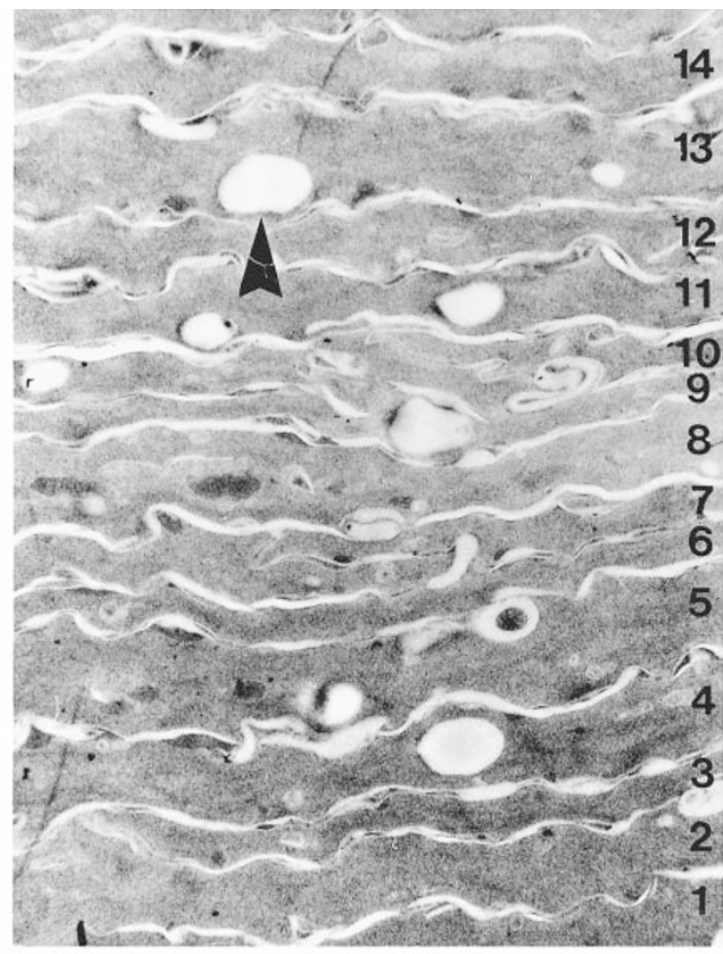

$\mathbf{C}^{\prime}$

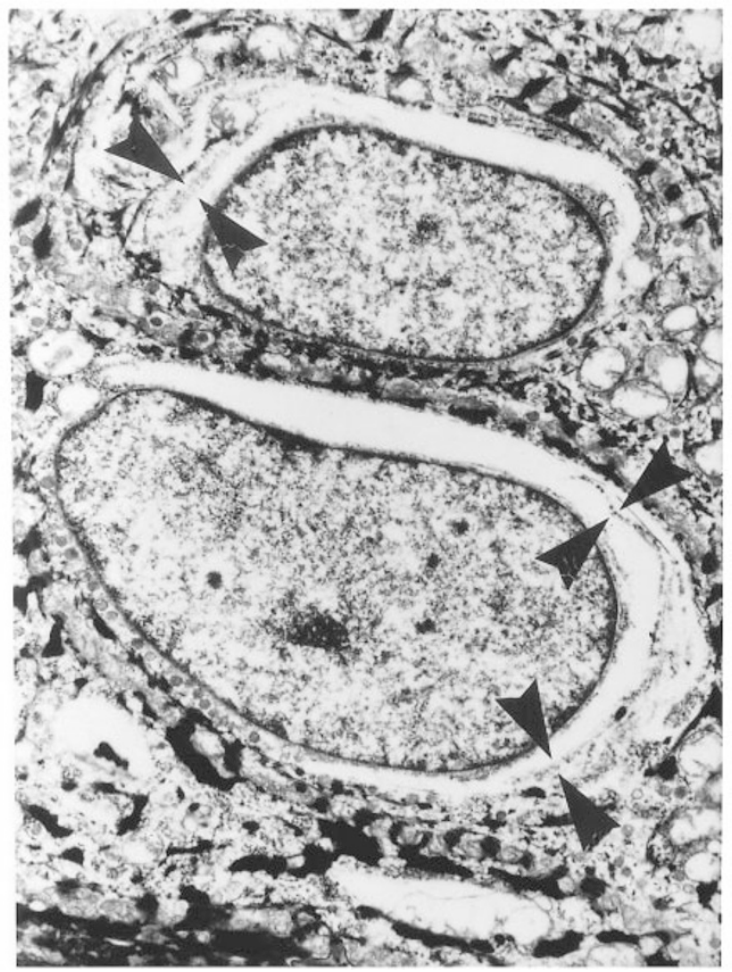

B

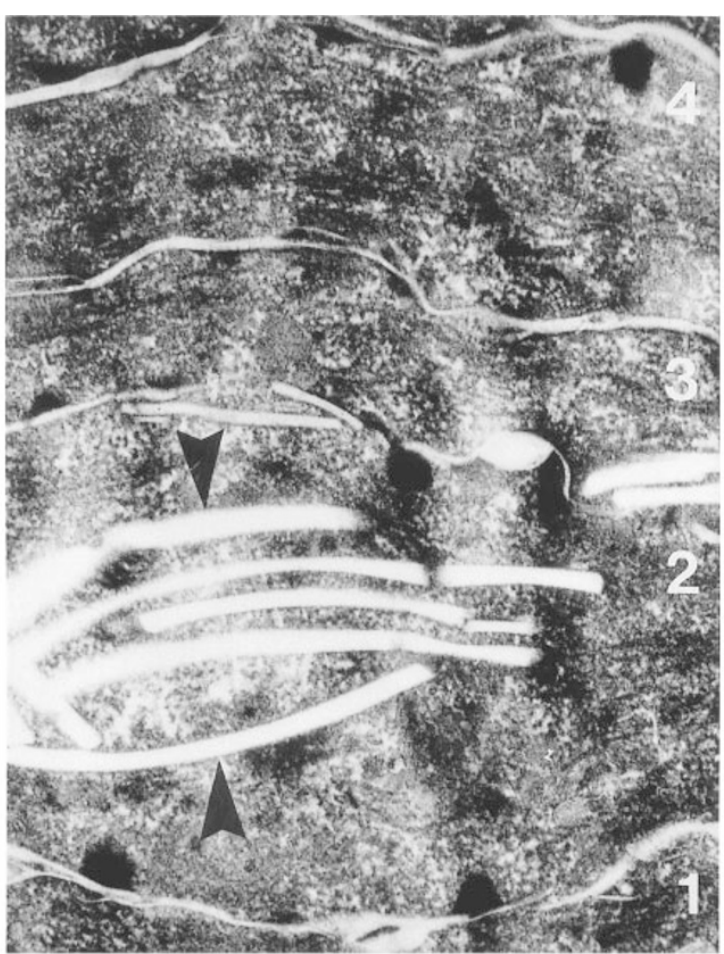

D

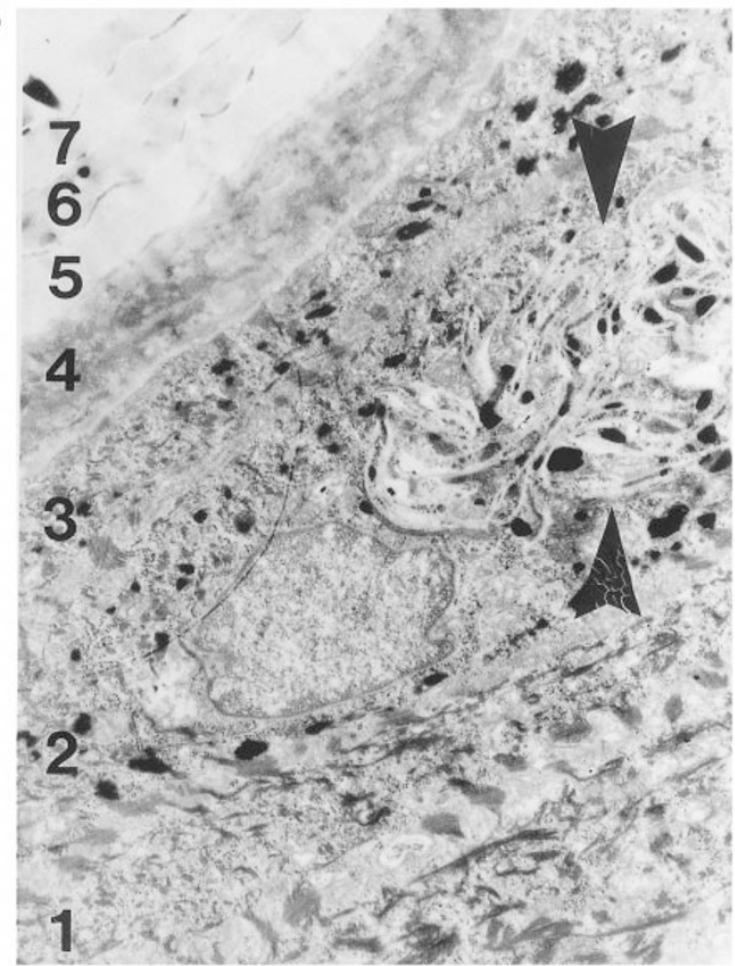

Figure 1 Electron microscopic findings of the four ichthyosis congenita types. a) A patient with IC type I (patient F6/P2 in Table 1): 14 horny cell layers numbered 1-14 are present with round lipid droplets (arrowhead). b) IC type II (patient F4/P2 in Table 1). Four keratin cell layers are numbered 1-4. Inside one of the cells there is a package of crystalloid structures called cholesterol clefts (between two arrowheads). c) IC type III. Two nuclei of two granular cells surrounded by elongated membrane structures (between three pairs of arrowheads). d) Patient of IC type IV: Three layers of the granular cells (numbered 1-3) and above them four horny cells (numbered 4-7). One of the granular cells with a package of membrane structures has been 'blown up' (between arrowheads) 
1983-1994, before genetic studies had been initiated. Part of the findings have been published previously. ${ }^{12-15}$ Of the 49 patients, 15 were under retinoid treatment when electronmicroscopic diagnosis was made.

\section{Molecular Genetic Studies}

All 49 patients were studied for TGM 1 mutations using single stranded conformation polymorphism analysis (SSCP) and sequencing as described elsewhere. ${ }^{11}$ After our previous studies one additional mutation, 3349A to $G$, was found in restriction enzyme Msp I analysis of 27 families as described earlier. $^{16}$

\section{Study of the Phenotype of the ARCI Patients}

The clinical picture was studied at the Department of Dermatology. Follow-up time for the patients varied from 1 to 30 years. Patients treated with retinoids were studied both before and after the retinoid treatment. Either of the oral retinoids Tigason (etretinate) or Neotigason (acitretin) was used (10-50 mg/day).

\section{Results}

\section{Ultrastructural Classification of Patients}

Electron microscopy was used in the classification of ARCI patients. In 15 families 23 patients were found to belong to IC type I, 10 patients from eight families to IC type II, six patients from six families to IC type III, and two patients from two families to IC type IV; eight patients from seven families could not be classified using electron microscopy in spite of their clearly ichthyotic skin (Figure 2). In every non-defined patient there was at least one exceptional finding in the electron microscopy, such as numerous vesicular structures or granular lysosomes which prevented assignment to any of the four IC types.

\section{TGM 1 Mutation versus Morphological IC Type}

The majority of the TGM 1 mutations were found in the IC type II category; eight families (62\%) of 13 TGM 1 mutation-carrying families were classified as type II. Correspondingly, all patients classified to IC type II were found to carry TGM1 mutations (Figure 2), (Table 1). The mutation profile in this group, however, was not homogeneous. Despite an identical clinical picture, the patients carried five different mutation combinations: Arg-142-Cys/Arg-142-Cys, Arg142-Cys/Val-378-Leu, Arg-141-His/Val-378-Leu, Val-378-Leu/Arg-395-Leu and Gly-217-Ser/Arg395-Leu.

TGM1 mutations were found also in patients classified into IC type I: five families (38\%) of 13 TGM 1 mutation carriers were classified as IC type I, each of the families having a different combination of mutations. No TGM1 mutations were detected in the patients of IC type III, IV or the non-defined group.

\section{Type of TGM 1 Mutation versus Clinical Phenotype}

In all eight type II families with TGM 1 mutations the clinical picture corresponded to classical LI with large,

\section{8 families with ARCI}

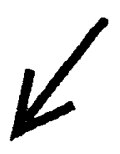

IC type I 15 families
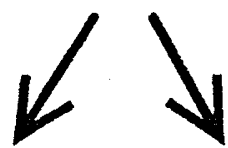

10 families,

5 families

No TGM 1 with TGM 1 mutation

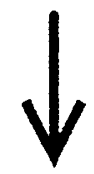

IC type II 8 families

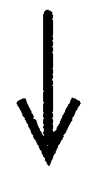

All 8 families No families with TGM 1 mutation

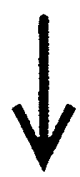

IC type III 6 families

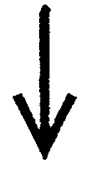

with TGM 1 mutation

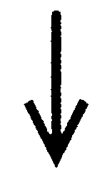

IC type IV 2 families

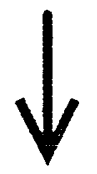

No families with TGM 1 mutation

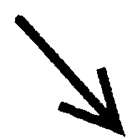

Non-defined group 7 families

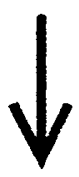

No families with TGM 1 mutation

Figure 2 Distribution of the TGM 1 mutations within different ichthyosis congenita types 
brownish scales in the skin (Table 1 ). However, in five of the 13 patients with TGM 1 mutations (originally classified as type I families) scaling of the skin was milder than in classic LI. Examples of clinical phenotype of type I and II patients with TGM 1 mutations are presented in Figure 3.

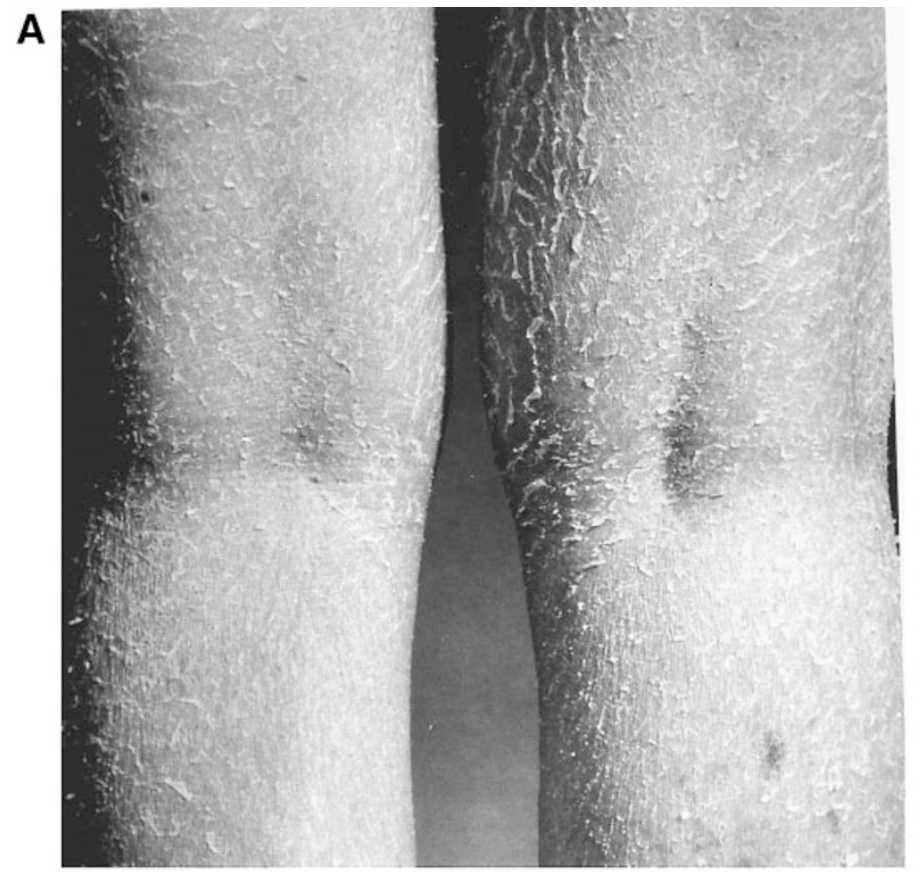

B

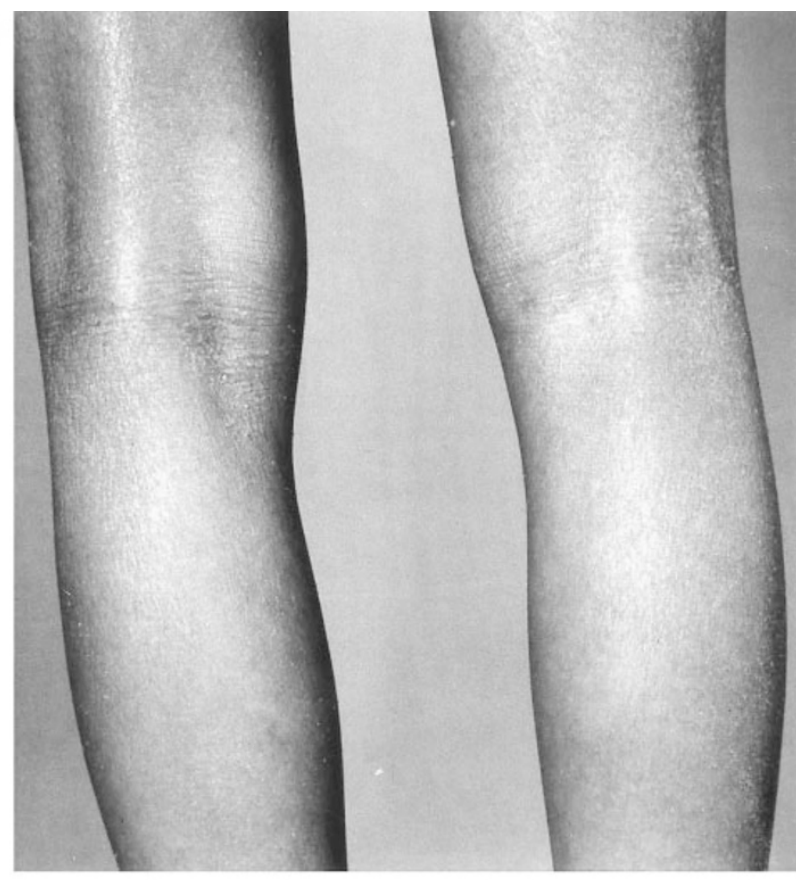

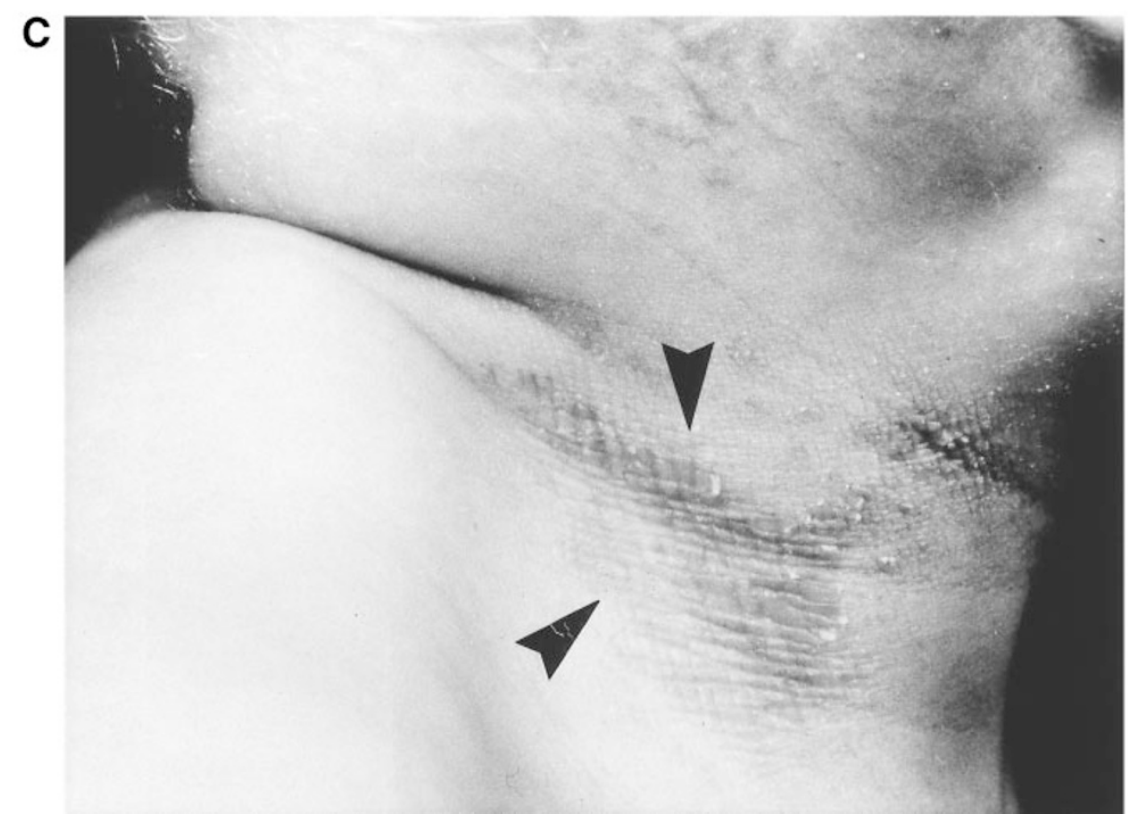

Figure 3 Examples of phenotypes of TGM1 gene mutation carriers. Both patients were treated with retinoids at the time of photographing. a) Clinical picture of the IC type II patient (patient F3/PII-4 in Table 1), carrier of Arg-142-Cys/Val-378-Leu mutation. b) Clinical picture of the IC type I patient (patient F6/P2 in Table 1), also a carrier of Arg-142-Cys/Val-378-Leu mutation. c) Clinical picture of an untypical patient. Note the patchy lesion in the neck (arrowheads). The rest of the skin is relatively normal (patient F8/P1 in Table 1, carrier of the Arg-142-Cys/3349 A to G) 
also 10 patients without TGM 1 mutations were born as collodion babies (part of them shown in Table1). Ectropion was present in all patients but one with TGM 1 mutations, whereas five of the TGM 1 mutation negative patients (not shown) had also ectropion. One IC type I patient (F8/P1, Table 1) was exceptional in many ways. Her skin was only partly ichthyotic (Figure 3c); she had thick scales only in the temporal region and on the right side of the trunk skin. Otherwise the skin was normal. She carried an Arg-142-Cys mutation in one allele and 3349A to $\mathrm{G}$ in the other allele.

\section{Discussion}

In this study we demonstrate that TGM1 mutations associate with IC types I and II. Electron-microscopic classification of ARCI seems to differentiate patients with a TGM 1 gene defect at least to some extent and might thus reflect the pathological process induced by the lack of cross-linking TGase 1 enzyme activity. Association of patients with TGM1 mutations to IC type II was not surprising as IC type II corresponds roughly to classic LI known to be caused by TGM 1 gene defects. ${ }^{4,5}$ Furthermore, as IC type I is clinically more heterogeneous it was not surprising to find five patients carrying TGM 1 mutations. Interestingly, none of the patients belonging to IC types III, IV or the nonclassified group carried a TGM 1 gene defect. Both of the IC types III and IV represent electron microscopically better delineated entities than IC type I. The finding of parallel electron microscopic and genetic groups may be useful for a further classification of patients for linkage studies attempting to find new genes underlying ARCI and to understand the pathogenesis of ARCI in more detail. Similar kinds of result were also seen in the study of 43 Norwegian ARCI families where one major founder mutation A2526G (corresponding to our mutation 3349 A to $G$ ) was found in patients with both type I and type II. ${ }^{16}$ Interestingly, this mutation was found only in one Finnish compound heterozygote patient whose clinical picture was quite untypical compared with other TGM 1 mutation carriers (Figure 3c).

This report is one of the first attempts to correlate the genotype with clinical phenotype and EM findings in ARCI patients. In our material most TGM1 mutationpositive patients had large, thick and brownish scales, consistent with the clinical diagnosis LI, but in five IC type I patients scales were thinner and ichthyosis not as severe as in type II. Although there was variation in the severity of ichthyosis within TGM1 mutation-positive patients, the patients with very mild ichthyosis and very thin fine scaling appeared not to have TGM1 mutations. No particular combination of mutations seemed to correlate with a particular IC type. Collodion baby syndrome and ectropion were found both in patients with TGM1 mutation and patients without TGM1 mutation. Results supporting diversity in the clinical picture within TGM1 mutation-positive patients were also found in the study of 14 German and Moroccan LI families. ${ }^{17}$

One could argue that retinoid treatment at the time of the electron microscopic diagnosis interferes with the classification and causes IC type II patients to become diagnosed as IC type I. However, it has been demonstrated that electron microscopic markers are not influenced by retinoid treatment. ${ }^{18,19}$ Also most of the patients were followed at the Department of Dermatology both before and after retinoid treatment.

One possible explanation for the clinical heterogeneity in recessive diseases is the type of mutation within the gene. Large deletions, insertions or mutations in the critical areas of the gene in general cause complete loss of the protein function and thus more severe clinical findings, whereas point mutations and mutations in less important areas may result in a milder course of the disease. Common aminoacid polymorphisms exist also in transglutaminases. ${ }^{20}$ In our ARCI patients all mutations but one are missense mutations, although in areas critical for the function of the enzyme, and in most cases patients are compound heterozygotes. Yet this kind of variability in clinical presentation, in spite of the similar genetic background, seems not to be infrequent. ${ }^{21}$ It is likely that most if not all of our patients' TGM1 mutations result in a dramatically reduced enzyme activity. This has been predicted based in both protein modelling and in vitro expression experiments. ${ }^{11,22,23}$

One explanation for the heterogeneity of the clinical picture can lie in the cornified cell envelope (CE) or in the expression of other genes producing attempts for compensatory mechanisms to overcome the lack of TGase 1. Defects in the TGase 1 enzyme are supposed to result in defective CE. However, the TGase 1 enzyme has been shown to exist in several intact or proteolytically processed cytosolic or membraneanchored forms which have different enzyme activities. $^{24}$ Because of the complexity of the TGase 1 
enzyme and the existence of large amounts of membrane-bound zymogen formed without transglutaminase activity, it is possible that TGM 1 has yet unidentified functions in cells. Cornified cell envelope is also crucial for the barrier function of the skin and altered barrier function can also have an effect on a number of other genes.

In conclusion, we demonstrate that TGM 1 mutations are associated with electron-microscopic IC types I and II. We also show that most TGM 1 mutation-positive patients have classic lamellar ichthyosis, but also patients with milder ichthyosis can have TGM 1 gene defects. Electron microscopic findings seem to follow molecular genetic alterations and this finding can be utilized in further linkage studies of ARCI.

\section{Acknowledgements}

We address special thanks, for technical assistance, to Eija Hämäläinen, Sinikka Laine, Tuula Salmivaara, Mervi Eeva, Maritta Putkiranta and Elina Honkavaara. We also thank the families for their excellent co-operation. This work was supported by the Sigrid Juselius Foundation and the Pediatric Research foundation, the Ulla Hjelt fund.

\section{References}

1 Williams ML, Elias PM: Heterogeneity in autosomal recessive ichthyosis: clinical and biochemical differentiation in lamellar ichthyosis and non-bullous congenital ichthyosiform erythroderma. Arch Dermatol 1985; 121: 477-488.

2 Williams ML, Elias PM: From basket weave to barrier. Arch Dermatol 1993; 129: 626-629.

3 Anton-Lamprecht I: The Skin. In: Papadimitriou JM, Henderson DW, Spagnolo DV (eds). Diagnostic Ultrastructure of non-Neoplastic Diseases, 1st edn. Churchill Livingstone: Edinburgh, 1992, pp 460-468.

4 Russell LJ, DiGiovanna JJ, Rogers GR et al: Mutations in the gene for transglutaminase 1 in autosomal recessive lamellar ichthyosis. Nat Genet 1995; 9: 279-283.

5 Huber M, Rettler I, Bernasconi K et al: Mutations of keratinocyte transglutaminase in lamellar ichthyosis. Science 1995; 267: 525-528.

6 Parmentier L, Lakhdar H, Blanchet-Bardon C, Marchand S, Dubertret L, Weissenbach J: Mapping of a second locus for lamellar ichthyosis to chromosome 2q33-35. Hum Mol Genet 1996; 5: 555-559.

7 Brody I: The modified plasma membranes of the transition and horny cells in normal human epidermis as revealed by electron-microscopy. Acta Derm Venereol 1969; 49: $128-138$
8 Steinert PM, Marekov L: Direct evidence that involucrin is a major early isopeptide cross-linked component of the keratinocyte cornified cell envelope. J Biol Chem 1997; 272: 2021-2030.

9 Goldman RD, Khuon S, Chou YH, Opal P, Steinert PM: The function of intermediate filaments in cell shape and cytoskeletal integrity. J Cell Biol 1996; 134: 971-983.

10 Hohl D: Cornified cell envelope. Dermatologica 1990; 180: 201-211.

11 Laiho E, Ignatius J, Mikkola $\mathrm{H}$ et al: Transglutaminase 1 mutations in autosomal recessive congenital ichthyosis: private and recurrent mutations in an isolated population. Am J Hum Genet 1997; 61: 529-538.

12 Niemi K-M, Kanerva L, Kuokkanen K, Ignatius J: Clinical, light and electron-microscopic features of recessive congenital ichthyosis type I. Br J Dermatol 1994; 130: 626-633.

13 Niemi K-M, Kanerva L, Kuokkanen K: Recessive ichthyosis congenita type II. Arch Dermatol Res 1991; 283 211-218.

14 Niemi K-M, Kanerva L, Wahlgren C-F, Ignatius J: Clinical, light and electron-microscopic features of recessive ichthyosis congenita type III. Arch Dermatol Res 1992; 284 259-265.

15 Niemi K-M, Kuokkanen K, Kanerva L, Ignatius J: Recessive ichthyosis congenita type IV. Am J Dermatopathol 1993; 15: 224-228.

16 Pigg M, Gedde-Dahl Jr T, Cox D, Haußer I, AntonLamprecht I, Dahl N: Strong founder effect for a transglutaminase 1 gene mutation in lamellar ichthyosis and congenital ichthyosiform erythromderma from Norway. Eur J Hum Genet 1998; 6: 589-596.

17 Hennies HC, Küster W, Wiebe V, Krebsovà A, Reis A: Genotype/phenotype correlation in autosomal recessive lamellar ichthyosis. Am J Hum Genet 1998; 62 1052-1061.

18 Kanerva L, Lauharanta J, Lassus A, Niemi K-M: New observations on the fine structure of lamellar ichthyosis and the effect of treatment with etretinate. Am J Dermatopathol 1983; 5: 555-567.

19 Marks R, Finlay AY, Holt PJA: Severe disorders of keratinization: effects of treatment with Tigason (etretinate). Br J Dermatol 1980; 104: 667-673.

20 Kim I-G, McBride W, Wang M, Kim S-Y, Idler WW, Steinert PM: Structure and organization of the human transglutaminase 1 gene. $J$ Biol Chem 1992; 267: 7710-7717.

21 Qian F, Germino GG: SOMATIC GENETICS '97 'Mistakes happen': Somatic mutation and disease. Am J Hum Genet 1997; 61: 1000-1005.

22 Huber M, Yee VC, Burri N et al: Consequences of seven novel mutations on the expression and structure of keratinocyte transglutaminase. J Biol Chem 1997; 272: 21018-21026.

23 Candi E, Melino G, Lahm A et al: Transglutaminase 1 mutations in lamellar ichthyosis. J Biol Chem 1998; 273 : 13693-13702.

24 Steinert PM, Kim S-Y, Chung S-Y, Marekov LN: The transglutaminase 1 enzyme is variably acylated by myristate and palmitate during differentiation in epidermal keratinocytes. J Biol Chem 1996; 271: 26242-26250. 